\title{
INSTRUMENTED INDENTATION FOR DETERMINATION OF FULL RANGE STRESS-STRAIN CURVES
}

\author{
A. De Smedt ${ }^{1}$, S. Hertelé ${ }^{2}$, M. Verstraete ${ }^{2}$, K. Van Minnebruggen ${ }^{2}$ and W. De Waele ${ }^{2}$ \\ ${ }^{1}$ Ghent University, Belgium \\ ${ }^{2}$ Ghent University, Laboratory Soete, Belgium
}

\begin{abstract}
One common method for the determination of full range stress-strain curves by instrumented indentation is presented and validated for an aluminium alloy. This method relates properties describing the indentation force-depth curve with those describing the uniaxial stress-strain curve as traditionally obtained from a tensile test. The first aim of this paper is to explain the basic concepts of instrumented indentation. Next, the analysis method is presented and validated. This study ends with discussing the uniqueness of the obtained solution. It is concluded that accurate determination of stress-strain behaviour can be realized, but for certain materials two indentations are needed.
\end{abstract}

Keywords: instrumented indentation, indenter, stress-strain, mystical materials

\section{INTRODUCTION}

Imagine one wants to obtain the local stress-strain properties of a metal in a non-destructive manner and with no restriction on specimen size and shape. This is made possible using an instrumented indentation test (IIT). In this paper the stress-strain curve of an aluminium alloy 6061-T6511 will be reconstructed by IIT $[1,2]$. This alloy is widely used in commercial construction applications.

IIT is a hardness test during which execution the force on the indenter and the displacement of the indenter are recorded. After specific data analysis, this results in a force-depth curve (Figure 1). This curve is characterised by a number of parameters that are discussed in section 2. The stress-strain curve of a metal is typically characterised by its Young's modulus, strain hardening exponent and yield strength. This will be elaborated in section 3. Several methods have been proposed to relate the parameters of an indentation force-depth curve with those of a stress-strain curve. Some brief remarks are given in section 4 . The curve fitting method used in this paper is fairly generic and widely used for the reconstruction of stress-strain curves. This is the topic of section 5. In section 6 the uniqueness of the obtained solution is discussed.
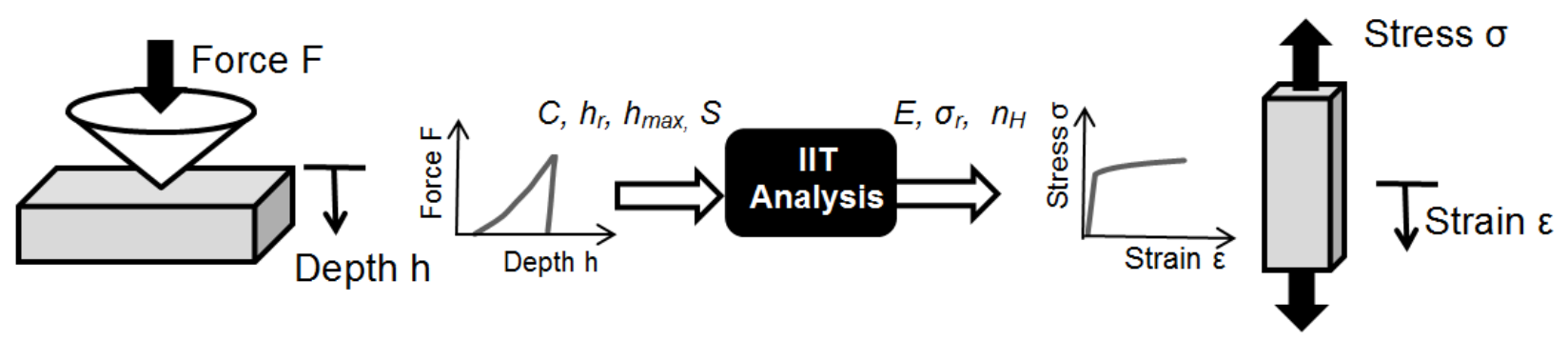

Figure 1: Concept of IIT

\section{INDENTATION FORCE-DEPTH CURVE}

The evolution of an indentation force-depth curve depends on the indenter type used but generally follows a characteristic pattern. This pattern is explained by means of an example, shown in Figure 2, obtained for the aluminium alloy 6061-T6511 using a Berkovich type indenter [2]. Such an indenter has the same projected area as a Vickers indenter (or a cone with a half tip angle of $70.3^{\circ}$ ) but is characterized by three facets instead of four. 


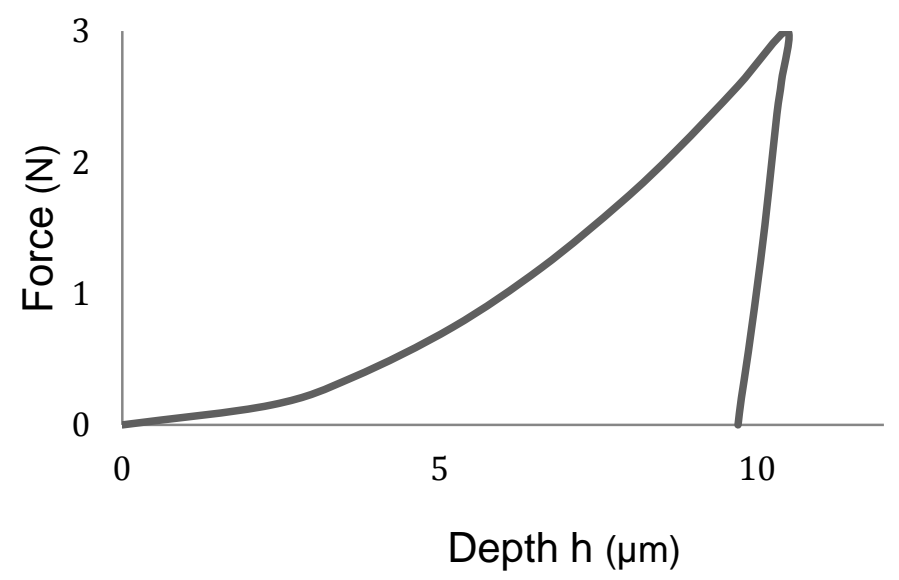

Figure 2: Indentation force-depth curve for an aluminium alloy 6061T6511 obtained using a Berkovich type indenter

The loading phase of the indentation response is described by Kick's law:

$$
P=C \cdot h^{2}
$$

For this specimen Kick's constant $C$ is $27.6 \mathrm{GPa}$. The (un)loading rate and maximum load are user defined and for the sample considered, these parameters are $4.4 \mathrm{~N} / \mathrm{min}$ and $3 \mathrm{~N}$ respectively. At the moment of maximum load, the maximum imprint depth $h_{\max }$ equals $10.19 \mu \mathrm{m}$.

Afterwards the material is unloaded, the indenter removed and the imprint recovers elastically. The unloading phase can be approximated by Eq. (2) in which $B, m$ and $h_{r}$ are curve fitting parameters [3].

$$
P=B \cdot\left(h-h_{r}\right)^{m}
$$

The unloading stiffness $S$ is defined as the slope of the unloading curve near the maximum indentation depth. It can be calculated by differentiating Eq. (2) and evaluating at $h_{\max }$ :

$$
S=\left.\frac{d P}{d h}\right|_{h_{\max }}=B \cdot m \cdot\left(h_{\max }-h_{r}\right)^{m-1}=4491 \mathrm{kN} / \mathrm{m}
$$

Much of the information obtained by one single instrumented indentation test is described by the four parameters introduced above: $h_{r}, h_{\max }, C$ and $S$. For some IIT methods the work performed during loading $W_{T}$ (area underneath the indentation force-depth curve during the loading phase) and unloading $W_{E}$ (area underneath the indentation force-depth curve during the unloading phase) are required rather than $h_{r}$ and $h_{\max }$. For sharp indenter types, $W_{T}$ and $W_{E}$ contain the same information as $h_{r}$ and $h_{\max }$ and are less sensitive to scatter because they are calculated based on all measured data points rather than a single value.

\section{STRESS-STRAIN CURVE}

The linear-elastic region of a stress-strain curve is characterized by the Young's modulus E. Further, the Poisson coefficient $v$ is introduced to describe lateral restraint effects during elastic (un)loading.

In instrumented indentation of metals, the stress-strain curve in the plastic area (i.e. stress exceeds the yield strength $\sigma_{Y}$ ) is typically approximated by a Hollomon type equation [4]:

$$
\sigma=R \cdot \epsilon^{n_{H}} \text { for } \sigma \geq \sigma_{Y}
$$

With $R$ the strength coefficient and $n_{H}$ the strain hardening exponent. The stress in the plastic area can be rewritten by Eq. (5) when elastic strain is given by $\varepsilon_{y}$, and the plastic component of strain by $\varepsilon_{p}$. 


$$
\sigma=R \cdot\left(\epsilon_{y}+\epsilon_{p}\right)^{n_{H}}=\sigma_{y}\left(1+\frac{\epsilon_{p}}{\epsilon_{y}}\right)^{n_{H}}=\sigma_{y}\left(1+\frac{E \cdot \epsilon_{p}}{\sigma_{y}}\right)^{n_{H}}
$$

This stress-strain model is described by $\left(E, \sigma_{y}, n_{H}\right)$ or $\left(E, \sigma_{r}, n_{H}\right)$ with $\sigma_{r}$ the 'representative' stress corresponding to a particular 'representative' strain as explained in section 5.

Figure 3 plots the true stress-strain curve of the aluminium alloy investigated in this paper determined by traditional tensile testing [2]. Its model parameters are $E=66.8 \mathrm{GPa}, n_{H}=0.8$ and $\sigma_{Y}=278 \mathrm{MPa}$.

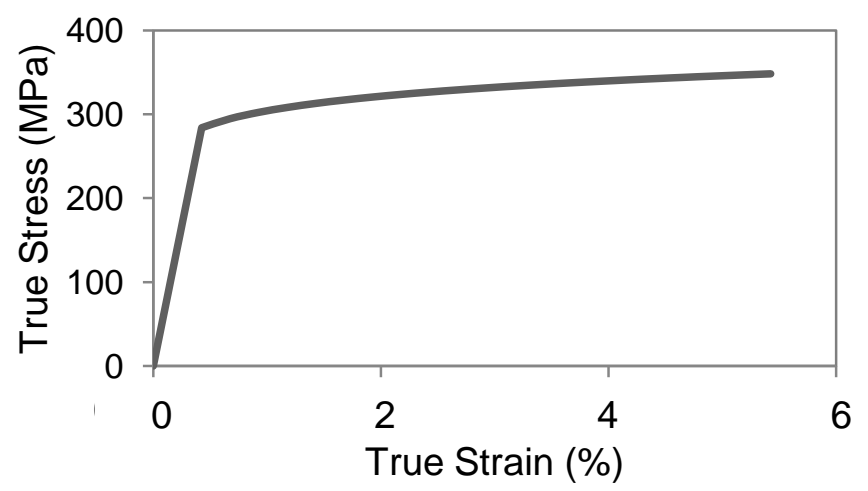

Figure 3: Stress-strain curve of the aluminium alloy 6061-T6511

\section{RELATIONSHIP BETWEEN INDENTATION FORCE-DEPTH AND STRESS-STRAIN CURVES}

A point-to-point systematic relationship between an indentation force-depth curve and a stress-strain curve would lead to a non-destructive method to determine the stress-strain properties of a steel. Such relationship has only been developed for spherical indentation (Brinell type indenter) [5-7], thereby making use of numerous profound assumptions and approximations. For sharp indentation (Vickers, Berkovich or Rockwell type indenters), however, finding an immediate link between both curves is highly challenging due to the geometrical similarity of this type of indentation [8]. This means that an IIT at large depth is essentially a magnified picture of an IIT at a small depth. As a consequence, methods based on a sharp indentation are confined to providing links between the model parameters of an indentation force-depth curve and a stress-strain curve rather than between their distinct data points.

The material in the vicinity of the indentation does not remain perfectly flat, which increases the complexity of characterising the deformation and developing adequate definitions for the representative stress and strain. Two different indentation responses may occur; pile-up and sink-in as illustrated on Figure 4.
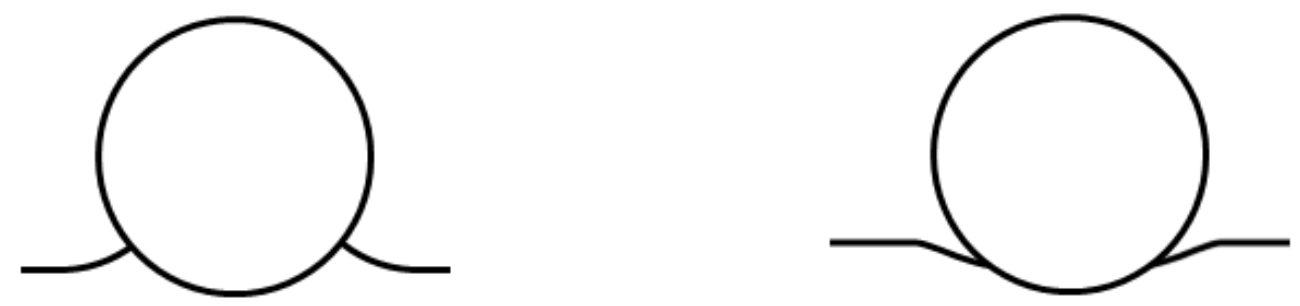

Figure 4: Pile-up (Left) and sink-in (right) influences the accuracy of stress and strain calculations

Multiple authors [5-7, 9] developed equations to predict pile-up and sink-in for certain material groups. The tendency towards pile-up increases with decreasing strain hardening exponent $n_{H}$ and $\sigma_{y} / E$ ratio [10]. Due to the many approximations which need to be made, a point-to-point relationship is limited in accuracy. 


\section{INSTRUMENTED INDENTATION ANALYSIS BY MEANS OF CURVE FITTING}

In curve fitting methods, the indentation parameters are written as functions of the unknown stress-strain properties. This enables the reconstruction of an indentation force-depth model curve out of the stressstrain model curve and vice versa. A selected curve fitting method for sharp indentation is applied to the indentation force-depth curve of Figure 2. This specific method is valid for common engineering materials: $E$ from 10 to $210 \mathrm{GPA}, \sigma_{\mathrm{Y}}$ from 30 to $3000 \mathrm{MPa}$ and $n_{H}$ from 0 to 0.5 , with Poisson's ratio fixed at 0.3 .

The influence of elastic deformations of both the indented metal and the indenter is captured by the reduced Young's modulus $E^{*}$ defined as:

$$
E^{*}=\left(\frac{1-v^{2}}{E}+\frac{1-v_{i}^{2}}{E_{i}}\right)^{-1}
$$

with $v_{i}$ the Poisson coefficient of the indenter and $E_{\mathrm{i}}$ its Young's modulus. For a diamond indenter these values equal 0.07 and $1140 \mathrm{GPa}$ respectively. The Young's modulus $E$ of the aluminium alloy is $66.8 \mathrm{GPa}$ and thus is the reduced Young's modulus $E^{*}$ equal to $70.4 \mathrm{GPa}$.

Next, three different universal dimensionless functions [11] are used to link both curves. Each author has its own functions but in general they have the same structure, given by the equations below [12-16].

$$
\begin{aligned}
& \prod_{1}\left(\frac{\mathrm{E}^{*}}{\sigma_{\mathrm{r}}}, \mathrm{n}\right)=\frac{\mathrm{C}}{\sigma_{\mathrm{r}}} \\
& \prod_{2}\left(\frac{\mathrm{E}^{*}}{\sigma_{\mathrm{r}}}, \mathrm{n}\right)=\frac{1}{\mathrm{E}^{*} \mathrm{~h}_{\mathrm{m}}} \mathrm{S} \\
& \prod_{3}\left(\frac{\mathrm{E}^{*}}{\sigma_{\mathrm{r}}}, \mathrm{n}\right)=\frac{\mathrm{h}_{\mathrm{r}}}{\mathrm{h}_{\mathrm{m}}}
\end{aligned}
$$

These functions are expressed as a function of the representative stress $\sigma_{r}$ corresponding with a plastic strain of 0.033 , rather than the yield strength $\sigma_{y}$. The choice of representative strain simplifies the solution to Eqs. (7)-(9), since with this value $\prod_{1}$ was empirically observed to be independent of $n_{H}$ (Figure 5). $\sigma_{r}$ is further also denoted as $\sigma_{0.033}$. By solving Eqs. (7), (8) and (9) the unknown material properties can be obtained. To illustrate this principle the functions will be solved graphically.

Given that the Young's modulus is known for aluminium alloys, only the Hollomon model parameters $\left(\mathrm{n}_{\mathrm{H}}\right.$ and $\sigma_{\mathrm{y}}$ ) of the stress-strain curve remain unknown. Thus only two dimensionless functions are needed to solve the problem. Because Le et al. [13] proved that Eq. (8) and Eq.(9) are dependent for sharp indentation, Eq. (7), Figure 5 and Eq. (8), Figure 6 are used to solve the problem.

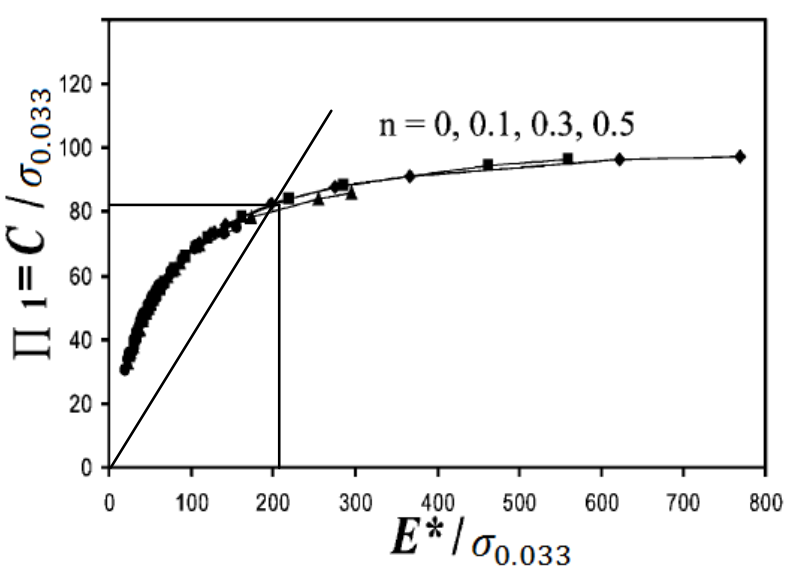

Figure 5: $\sigma_{\mathrm{r}}$ as a function of indentation parameters [11]

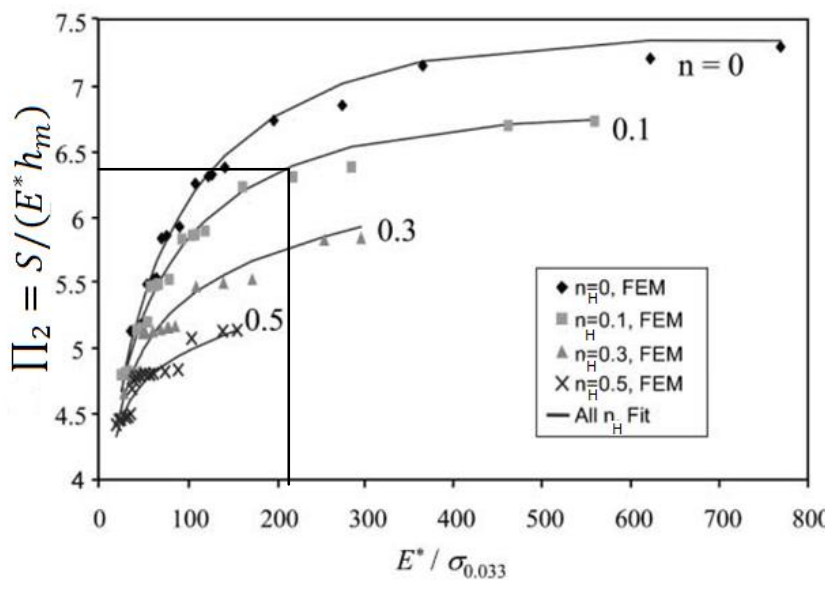

Figure 6: $n_{H}$ as a function of indentation parameters [11]

With $C$ and $E^{*}$ a priori known (27.6 GPa and $70.4 \mathrm{GPa}$ respectively) a straight line with slope equal to $C / E^{*}$ can be drawn on Figure 5 . From the intersection of this line with the function $\Pi_{1}$, the representative stress $\sigma_{0.033}$ is determined as $337.4 \mathrm{MPa}$. $S$ and $h_{\max }$ are $4500 \mathrm{kN} / \mathrm{m}$ and $10.19 \mu \mathrm{m}$ respectively and thus from 
Figure 6 it follows that $\mathrm{n}_{\mathrm{H}}=0.088$. The yield stress can be calculated using Eq. (5), resulting in $\sigma_{y}=278$ $\mathrm{MPa}$.

Figure 7 shows the resulting stress-strain curve which is in good approximation with the one obtained from the tensile test ( $10 \%$ error on $n_{H}$ and $2 \%$ error on $\sigma_{Y}$ ).

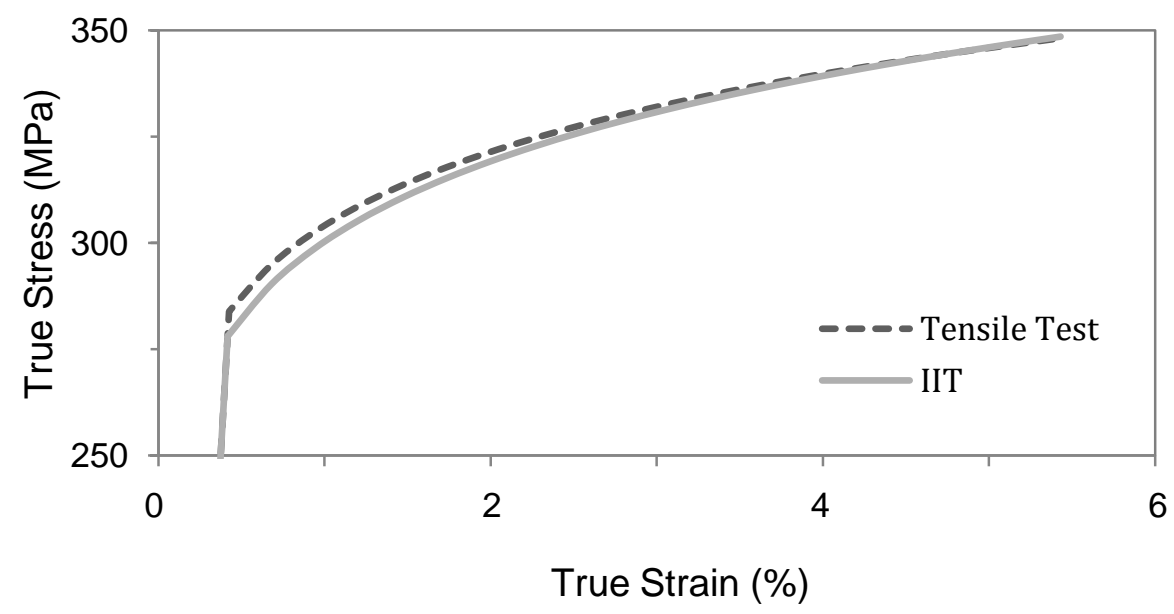

Figure 7: IIT has the potential to provide good approximations for stress-strain behaviour

\section{UNIQUENESS OF THE SOLUTION}

If the Young's modulus is not known in advance, it is impossible to reconstruct the stress-strain curve uniquely by sharp indentation, given the dependence of Eqs. (8) and (9). It is clear from Figure 8 that different materials may yield the same indentation force-depth curve for sharp indentation, independent of the dimensionless relations used. Such materials are referred to as 'mystical materials'. By using a second and different indenter (e.g. a $60^{\circ}$ cone, see Figure 8) in a so called 'dual indenter method', different materials are uniquely distinguishable. Several dual indenter methods exist $[2,13,17-20]$ but with other dimensionless functions than Eqs. (7)-(9). Apart from adopting a second indenter, increasing the penetration depth decreases the likeliness that the force-depth curve is the same for the different materials. However, with increasing penetration depth, unpredictable and therefore undesired frictional effects become increasingly pronounced.

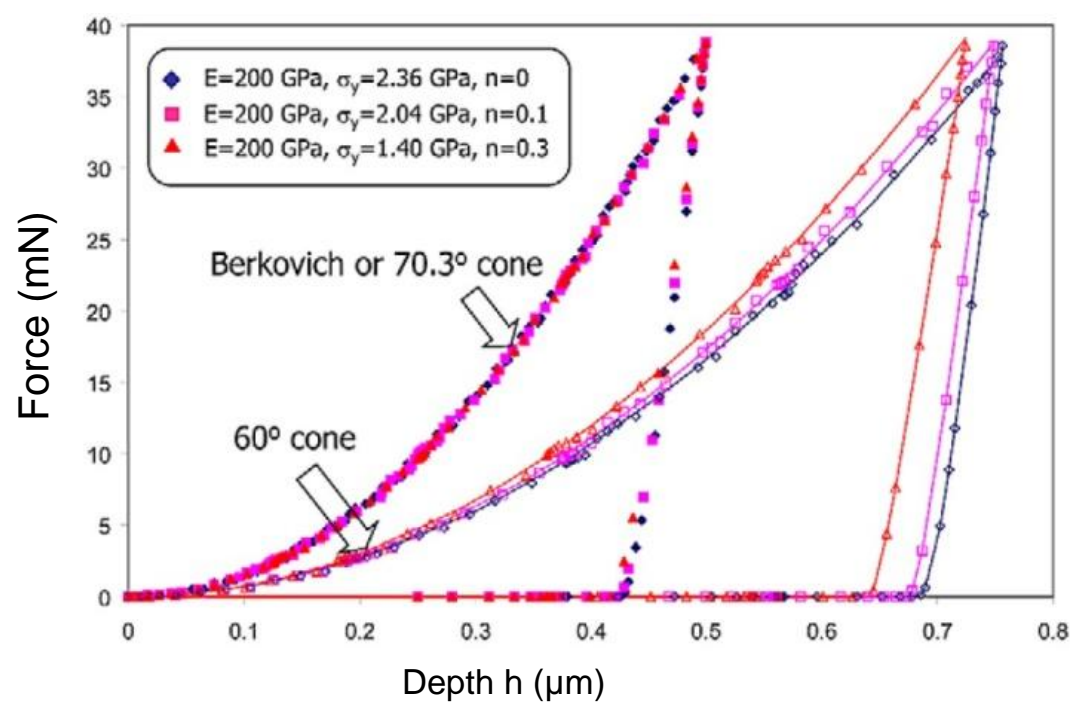

Figure 8: Impossibility to uniquely reconstruct the stress-strain curve with only one Berkovich indenter [2] 


\section{CONCLUSION}

Instrumented indentation allows for the construction of uniaxial stress-strain curves using the recorded indentation force-depth curve as an input. This paper has illustrated a common method, based on curve fitting and applied for sharp indenters. A case study indicates that satisfactory results can be obtained. Transforming an indentation force-depth curve into a uniaxial stress-strain curve is challenging due to poorly quantifiable phenomena such as pile-up and sink-in, and the existence of mystical materials. It is shown that for uniquely determining the stress-strain properties two indentations with different indenters may be necessary. Both indentations can then be analysed using the single indentation method explained in this paper. This leads to a sufficient number of independent equations to solve for the stress-strain curve parameters.

\section{ACKNOWLEDGEMENTS}

The authors would like to acknowledge the advice of Prof. Didier Chicot from Université De Lille 1.

\section{REFERENCES}

[1] Aluminium standards and data 2006 metric SI, the aluminium Association Inc.

[2] Chollacoop, N., M. Dao, and S. Suresh, Depth-sensing instrumented indentation with dual sharp indenters. Acta Materialia, 2003. 51(13): p. 3713-3729.

[3] Luo, J. and J. Lin, A study on the determination of plastic properties of metals by instrumented indentation using two sharp indenters. International Journal of Solids and Structures, 2007. 44(1819): p. 5803-5817.

[4] Hollomon, J.H., Tensile deformation. Transactions of the AIME. 1945: p. 162-268, 290.

[5] Collin, J.-M., G. Mauvoisin, and P. Pilvin, Materials characterization by instrumented indentation using two different approaches. Materials \& Design, 2010. 31(1): p. 636-640.

[6] Kim, J.-Y., et al., Determination of tensile properties by instrumented indentation technique: Representative stress and strain approach. Surface and Coatings Technology, 2006. 201(7): p. 4278-4283.

[7] Lee, K.-W., et al., Safety assessment based on mapping of degraded mechanical properties of materials for power plant pipeline using instrumented indentation technique. Journal of Loss Prevention in the Process Industries, 2009. 22(6): p. 868-872.

[8] Tabor, D., The hardness of solids. Review of physics in technology, 1970. 1: p. 145-178.

[9] Collin, J.M., G. Mauvoisin, and R. El Abdi, An experimental method to determine the contact radius changes during a spherical instrumented indentation. Mechanics of Materials, 2008. 40(4-5): p. 401-406.

[10] Ma, Z.S., et al., Characterization of stress-strain relationships of elastoplastic materials: An improved method with conical and pyramidal indenters. Mechanics of Materials, 2012. 54(0): p. 113-123.

[11] Dao, M., et al., Computational modeling of the forward and reverse problems in instrumented sharp indentation. Acta Materialia, 2001. 49(19): p. 3899-3918.

[12] Cao, Y., X. Qian, and N. Huber, Spherical indentation into elastoplastic materials: Indentationresponse based definitions of the representative strain. Materials Science and Engineering: $A$, 2007. 454-455(0): p. 1-13.

[13] Le, M.-Q., A computational study on the instrumented sharp indentations with dual indenters. International Journal of Solids and Structures, 2008. 45(10): p. 2818-2835.

[14] Le, M.-Q., Material characterization by instrumented spherical indentation. Mechanics of Materials, 2012. 46(0): p. $42-56$.

[15] Ogasawara, N., N. Chiba, and X. Chen, A simple framework of spherical indentation for measuring elastoplastic properties. Mechanics of Materials, 2009. 41(9): p. 1025-1033.

[16] Tho, K.K., et al., Simulation of instrumented indentation and material characterization. Materials Science and Engineering: A, 2005. 390(1-2): p. 202-209.

[17] Bucaille, J.L., et al., Determination of plastic properties of metals by instrumented indentation using different sharp indenters. Acta Materialia, 2003. 51(6): p. 1663-1678.

[18] Le, M.-Q., Material characterization by dual sharp indenters. International Journal of Solids and Structures, 2009. 46(16): p. 2988-2998.

[19] Le, M.-Q., Improved reverse analysis for material characterization with dual sharp indenters. International Journal of Solids and Structures, 2011. 48(10): p. 1600-1609.

[20] Swaddiwudhipong, S., et al., Material characterization based on dual indenters. International Journal of Solids and Structures, 2005. 42(1): p. 69-83. 